\title{
Biochemical and structural study on a S529V mutant acid $\alpha$-glucosidase responsive to pharmacological chaperones
}

\author{
Youichi Tajima $^{1,2,7}$, Seiji Saito ${ }^{3,7}$, Kazuki Ohno ${ }^{4,8}$, Takahiro Tsukimura ${ }^{5}$, Seiichi Tsujino ${ }^{6}$ and \\ Hitoshi Sakuraba ${ }^{1,5}$
}

Recently, pharmacological chaperone therapy for Pompe disease with small molecules such as imino sugars has attracted interest. But mutant acid $\alpha$-glucosidase (GAA) species responsive to imino sugars are limited. To elucidate the characteristics of a mutant GAA responsive to imino sugars, we performed biochemical and structural analyses. Among cultured fibroblast cell lines derived from Japanese Pompe patients, only one carrying p.S529V/p.S619R amino acid substitutions responded to 1-deoxynojirimycin (DNJ), and an expression study revealed that DNJ, $N$-butyl-deoxynojirimycin and nojirimycin-1-sulfonic acid increased the enzyme activity of the S529V mutant GAA expressed in Chinese hamster ovary cells. The results of western blotting analysis suggested that these imino sugars facilitated the intracellular transportation of the mutant GAA and stabilized it. Among these imino sugars, DNJ exhibited the strongest action on the mutant GAA. Structural analysis revealed that DNJ almost completely occupied the active site pocket, and interacted with amino acid residues comprising it through van der Waals contacts and hydrogen bonds. This information will be useful for improvement of pharmacological chaperone therapy for Pompe disease.

Journal of Human Genetics (2011) 56, 440-446; doi:10.1038/jhg.2011.36; published online 7 April 2011

Keywords: acid $\alpha$-glucosidase; imino sugar; pharmacological chaperone; Pompe disease; structure

\section{INTRODUCTION}

Acid $\alpha$-glucosidase (GAA, acid maltase; EC. 3.2.1.20/3) is an enzyme belonging to a group of family 31 glucosyl hydrolases that catalyze hydrolysis of the $\alpha$-1,4- and $\alpha$-1,6-glucosidic bonds of oligosaccharides and glycogen in lysosomes. The enzyme, encoded by the GAA gene located on chromosome $17 \mathrm{q} 25$, is synthesized as a preproprotein composed of 952 amino acid residues, transported into the lumen of the endoplasmic reticulum, and subsequently $N$-glycosylated, which results in a $110 \mathrm{kDa}$ precursor glycoprotein. Then, it undergoes further modification of its sugar chains in the endoplasmic reticulum and Golgi apparatus. Finally, it is transported to lysosomes, where it is modified into a $76 \mathrm{kDa}$ mature enzyme and a minor $70 \mathrm{kDa}$ component via a $95 \mathrm{kDa}$ intermediate. ${ }^{1-4}$

A genetic defect in the enzyme causes the accumulation of glycogen in lysosomes and an abnormality of autophagy, ${ }^{5}$ thereby leading to Pompe disease (glycogen storage disease type II; OMIM 232300). The disease exhibits a wide range of clinical phenotypes, from the earlyonset severe 'infantile form', which is characterized by cardiomegaly, hepatomegaly, hypotonia and muscular weakness, to the late-onset slowly progressive 'juvenile/adult form', which shows a predominance of muscular disorders. ${ }^{1}$

In Pompe disease, close to 300 disease-causing mutations have been identified, the majority of them being missense ones. ${ }^{1,6-8}$ The mutant enzymes having single amino acid substitutions, regardless of their activity competency, are misfolded and degraded by the endoplasmic reticulum's quality control system before being transported to lysosomes. 9

In use as therapy for Pompe disease, a recombinant human GAA produced in Chinese hamster ovary $(\mathrm{CHO})$ cells is available. ${ }^{10}$ Despite its efficacy, replacement therapy with this enzyme has some problems, that is, the high cost, and its low uptake by the brain and skeletal muscles. ${ }^{111}$ Recently, pharmacological chaperone therapy with small molecules has attracted interest. Low molecular weight chemicals are expected to selectively interact with mutated but enzymologically active proteins, stabilize them and facilitate their transport to lysosomes. ${ }^{12}$ As such, potential pharmacological chaperones for GAA,

\footnotetext{
${ }^{1}$ Department of Clinical Genetics, Meiji Pharmaceutical University, Tokyo, Japan; ${ }^{2}$ Department of Molecular Medical Research, The Tokyo Metropolitan Institute of Medical Science, Tokyo Metropolitan Organization for Medical Research, Tokyo, Japan; ${ }^{3}$ Department of Biotechnology, Graduate School of Agricultural and Life Science, The University of Tokyo, Tokyo, Japan; ${ }^{4}$ NPO for the Promotion of Research on Intellectual Property Tokyo, Tokyo, Japan; ${ }^{5}$ Department of Analytical Biochemistry, Meiji Pharmaceutical University, Tokyo, Japan and ${ }^{6}$ Department of Peripheral Nervous System Research, National Institute of Neuroscience, NCNP, Tokyo, Japan

${ }^{7}$ These authors contributed equally to this work.

${ }^{8}$ Current address: Drug Discovery Research, Astellas Pharma Inc., 21 Miyukigaoka, Tsukuba, Ibaraki, 305-8585, Japan.

Correspondence: Dr H Sakuraba, Department of Analytical Biochemistry, Meiji Pharmaceutical University, 2-522-1 Noshio, Kiyose, Tokyo 204-8588, Japan.

E-mail: sakuraba@my-pharm.ac.jp
}

Received 18 November 2010; revised 17 February 2011; accepted 4 March 2011; published online 7 April 2011 
imino sugars including 1-deoxynojirimycin (DNJ) and N-butyl-deoxynojirimycin (NB-DNJ) are known. ${ }^{13,14}$ However, mutant GAAs responsive to these imino sugars are restricted to a limited group with specific amino acid substitutions, and the enhancing effects of the imino sugars available are not so strong. ${ }^{13-15}$ For further improvement of pharmacological chaperone therapy for Pompe disease, detailed information on mutant GAAs responsive to small molecules is required.

In this study, we examined the interaction of a mutant GAA with imino sugars, biochemically and structurally, and determined its characteristics.

\section{MATERIALS AND METHODS}

\section{Chemicals}

DNJ, NB-DNJ and nojirimycin-1-sulfonic acid (NSA) were obtained from Toronto Research Chemicals (North York, Ontario, Canada). 4-Methylumbelliferyl- $\alpha$-D-glucopyranoside was purchased from Calbiochem (La Jolla, CA, USA). The compounds used were of reagent grade.

\section{Primary Pompe fibroblast cell lines and culture}

As a preliminary study, cultured fibroblast cell lines from 10 Japanese patients with Pompe disease (five infantile form patients and five late-onset ones) from different families ${ }^{6,16-18}$ were examined to identify the ones responsive to DNJ. Information on the cells is summarized in Table 1. The cells were treated for 7 days with or without $50 \mu \mathrm{M}$ DNJ, and then GAA activity was determined. Among the cell lines, only F660 (phenotype, late-onset; genotype, p.S529V/ p.S619R) responded to DNJ. DNJ did not exhibit any effect on the activities of other lysosomal enzymes including $\alpha$-galactosidase A, $\beta$-galactosidase, $\beta$-glucosidase, $\alpha$-mannosidase and $\alpha$-fucosidase, under the conditions examined here (data not shown). Considering this finding, F660 was used as a sample for the following examinations. F664 (late-onset; p.R437C/p.R437C) and $\mathrm{Fw}$ (healthy; wild type/wild type) were used as pathological and normal controls, respectively. The study involving the cultured human fibroblasts was approved by the Ethics Committee of our institute.

The cells were cultured in Ham's F-10 medium containing 10\% fetal calf serum and antibiotics at $37^{\circ} \mathrm{C}$ in a humidified incubator flushed continuously with a $5 \% \mathrm{CO}_{2}-95 \%$ air mixture.

\section{Assaying of GAA and protein determination}

GAA activity was determined fluorometrically using 4-methylumbelliferyl- $\alpha$-Dglucopyranoside as a substrate, as described previously. ${ }^{19}$ Briefly, $10 \mu \mathrm{l}$ of a cell homogenate was added to $40 \mu \mathrm{l}$ of a substrate solution comprising $4 \mathrm{~mm}$ 4-methylumbelliferyl- $\alpha$-D-glucopyranoside in $0.2 \mathrm{M}$ citrate buffer, $\mathrm{pH}$ 4.0. The reaction mixture was incubated at $37^{\circ} \mathrm{C}$ for $30 \mathrm{~min}$, and then the reaction was stopped by the addition of $950 \mu \mathrm{l}$ of $0.2 \mathrm{M}$ glycine buffer, $\mathrm{pH}$ 10.7. Then the fluorescence intensity was measured with a Wallac 1420 ARVO MX multilabel

Table 1 Cultured fibroblasts derived from Japanese patients with Pompe disease

\begin{tabular}{lllc}
\hline Cells & Phenotype & Genotype & References \\
\hline F91 & Infantile & p.C103X/p.C103X & 6 \\
F239 & Infantile & Undefined & New case \\
F297 & Late-onset & Undefined & New case \\
F366 & Infantile & Undefined & New case \\
F415 & Late-onset & Undefined & New case \\
F659 & Infantile & p.M439K/undefined & 18 \\
F660 & Late-onset & p.S529V/p.S619R & 16,17 \\
F661 & Infantile & p.R600C/undefined & 18 \\
F663 & Late-onset & p.R608X/undefined & 18 \\
F664 & Late-onset & p.R437C/p.R437C & 17 \\
\hline
\end{tabular}

counter (Perkin-Elmer, Waltham, MA, USA) at excitation and emission wavelengths of 355 and $460 \mathrm{~nm}$, respectively. Protein concentrations were determined with a Micro BCA Protein Assay Reagent Kit (Thermo Scientific, Rockfold, IL, USA), bovine serum albumin being used as a standard.

\section{Examination of the effects of imino sugars on primary Pompe fibroblasts}

The cell lines F660, F664 and Fw were cultured for 7 days in medium containing various concentrations $(0,50,100$ and $300 \mu \mathrm{M})$ of DNJ, NB-DNJ or NSA. The cells were collected with trypsin-EDTA and washed twice with phosphate-buffered saline (PBS), and then homogenates of them were used for protein determination and GAA assaying.

\section{Preparation of mutant GAA constructs}

Human GAA cDNA (Open Biosystems, Huntsville, AL, USA) was used as a template. The coding region for the GAA was amplified by PCR using the following primers: sense, $5^{\prime}$-CCCAAGCTTATGGGAGTGAGGCACCCGCCC-3' and antisense, 5'-GGGAATTCCTAACACCAGCTGACGAGAAA-3', the italicized nucleotides being the HindIII and EcoRI sites, respectively. A PCR fragment of the coding region of GAA was digested with HindIII and EcoRI, and then introduced into the pEE14.4 expression vector (Lonza Biologic, Allendale, NJ, USA). Site-directed mutagenesis was performed as previously described. ${ }^{20}$ Then, the GAA construct described above was used as a template to prepare expression vectors for mutant GAAs. The following primer sets were used to generate expression vectors for S529V and S619R mutant GAAs, respectively: sense, $5^{\prime}$-CGTTGAGGACGGCTGCCCCAACAAT- $3^{\prime}$ and antisense, $5^{\prime}$-CCCCTGATGAAGTTGGAAGGCTCGT-3' for S529V; and sense, $5^{\prime}$-AGATC CTGGGAGCAGCTCGCCTCCT- ${ }^{\prime}$ and antisense, $5^{\prime}$-CCACACGTCCCCCGTC CAGTGGCCG-3' for S619R. The mutations were confirmed by nucleotide sequencing analysis.

\section{Generation of $\mathrm{CHO}$ cells expressing the wild type and mutant} GAAs, and determination of the effects of imino sugars on GAAs expressed in the cells

CHO-K1 cells were cultured in $\alpha$-modified Eagle's medium supplemented with $10 \%$ fetal bovine serum and antibiotics at $37^{\circ} \mathrm{C}$ in a humidified incubator flushed continuously with a $5 \% \mathrm{CO}_{2}-95 \%$ air mixture. Transfection was performed with $1 \mu \mathrm{g}$ plasmid expressing the S529V or S619R mutant GAA, or the wild-type one, as a control, per 12-well plate using Lipofectoamine LTX and Plus reagents (Invitrogen, Carlsbad, CA, USA) according to the manufacturer's instructions. Mock transfection was performed with the vector alone. After transfection, the cells were cultured for $5 \mathrm{~h}$ in the same medium, which was then replaced by medium containing various concentrations $(0,10,50$ and $300 \mu \mathrm{m})$ of DNJ, NB-DNJ or NSA. After $72 \mathrm{~h}$, the cells were collected with $0.125 \%$ trypsin-EDTA and washed with PBS, and then the cell pellets were sonicated in $50 \mathrm{~mm} 2$-( $\mathrm{N}$-morpholino) ethanesulfonic acid buffer, $\mathrm{pH}$ 6.0, as described previously. ${ }^{19}$ The cell homogenates were used for protein determination, GAA assaying and western blotting analysis.

\section{Western blotting analysis}

Ten-microgram aliquots of protein in the transiently transfected CHO-K1 cell extracts were each denatured in a one-fourth volume of $4 \times$ NuPAGE lithium dodesyl sulfate sample buffer $(424 \mathrm{~mm}$ Tris- $\mathrm{HCl}, \mathrm{pH} 8.5,292 \mathrm{~mm}$ lithium dodesyl sulfate, $4.36 \mathrm{~m}$ glycerol, $2 \mathrm{~mm}$ EDTA and $50 \mathrm{~mm}$ dithiothreitol) at $70^{\circ} \mathrm{C}$ for $10 \mathrm{~min}$. Then, they were separated on a Bis-Tris polyacrylamide gel (NuPAGE 4-12\%; Invitrogen), and electrotransferred to a polyvinylidene difluoride Immobilon-P membrane (Millipore Corporation, Bedford, MA, USA). For western blotting, each membrane was blocked with $5 \%$ non-fat skim milk in PBS/0.1\% Tween-20, and then incubated overnight with rabbit polyclonal antibodies to human GAA (1:500 diluted) in blocking buffer. ${ }^{19}$ Subsequently, the blot was washed in PBS/0.1\% Tween-20, and then reacted with peroxidase-conjugated donkey anti-rabbit secondary antibodies (GE Healthcare Bio-Sciences, Piscataway, NJ, USA) and an ECL plus Western Blotting Detection System (GE Healthcare) as a chemiluminescent substrate. 
The resulting bands were visualized with an LAS-4000 Luminescent Image Analyser (GE Healthcare)

\section{Structural modeling of mutant GAAs}

Structural models of the S529V and S619R mutant GAAs were built by means of homology modeling. At first, a structural model of the wild type human GAA was built with Molecular Operating Environment (CCG-Chemical Computing Group, Montreal, Quebec, Canada), using the crystal structure of the $N$-terminal subunit of human intestinal maltase-glucoamylase (PDB: 2QLY) as a template, followed by energy minimization, as described previously. ${ }^{21}$ The root mean square gradient value was set at $0.05 \mathrm{kcal} \mathrm{mol}^{-1} . \AA$. Then, structural models of the mutant GAAs were built with TINKER, ${ }^{22-23}$ using the modeled structure of the wild-type GAA as a template. ${ }^{21}$

\section{Calculation of numbers of atoms influenced by amino acid} substitutions

Each mutant model was superimposed on the wild-type GAA structure on the basis of the $\mathrm{C} \alpha$ atoms by the least-square mean fitting method. We defined that the structure was influenced by an amino acid substitution when the position of an atom in a mutant structure differed from that in the wild-type one by more than $0.15 \AA$, based on total root mean square distance, and the numbers of influenced atoms in the main chain and the side chain were calculated, as described previously. ${ }^{24}$

Determination of root mean square distance values of all atoms in the mutant GAA structures

The root mean square distance values of all atoms in the S529V and S619R mutant GAAs were determined according to Weiner's method, as described previously. ${ }^{24}$

Determination of solvent-accessible surface area (ASA) values of amino-acid residues in the mutant GAA structures

The ASA value of each residue in the wild-type GAA was calculated using ACCESS, ${ }^{25}$ and the position of each substituted amino acid residue in the S529V and S619R mutant GAAs was determined, as described previously. ${ }^{24}$

\section{Coloring of the atoms influenced by amino acid substitutions}

Coloring of the influenced atoms in the three-dimensional structures of the S529V and S619R mutant GAAs was performed for the corresponding amino acid substitutions as to the distance between the wild-type and mutant ones, as described previously. ${ }^{24}$
Structural modeling of the complex of the S529V mutant GAA with DNJ bound to its active site

Previously, we built a structural model of the catalytic domain of GAA by means of homology modeling using the structural information on $\alpha$-glucosidase MalA from Sulfolobus solfactrics (PDB: 2G3N), and predicted the structure of the wild type GAA/DNJ complex. ${ }^{26}$ In this study, we used a new structural model of the human GAA described above as a template, and predicted the structure of the complex between the mutant GAA and DNJ. AutoDock 4.0 (Molecular Graphics Laboratory, La Jolla, CA, USA), a grid-based docking program, was used to dock DNJ to the S529V mutant GAA according to the method described previously. ${ }^{27}$

\section{RESULTS}

Effects of imino sugars on GAA activity in primary Pompe fibroblasts

The effects of imino sugars including DNJ, NB-DNJ and NSA on GAA activity in cultured fibroblasts from two Japanese patients with Pompe disease harboring p.S529V/p.S619R and p.R432C/p.R437C, respectively, and a healthy control were examined. The results are shown in Figure 1.

DNJ treatment of F660 resulted in an apparent increase in endogenous GAA activity (fourfold increase with $50 \mu \mathrm{M} \mathrm{DNJ}$ ), and that of NSA a moderate one (twofold increase with $50 \mu \mathrm{m}$ NSA). The enhancement occurred dose-dependently under the experimental conditions used. NB-DNJ did not show any enhancement. This suggests that the S529V and/or S619R mutant GAA proteins respond to DNJ and NSA. The imino sugars did not cause any clear enhancement for F664. For Fw, both NB-DNJ and NSA rather inhibited it.

\section{Effects of imino sugars on the mutant GAAs expressed in CHO-K1 cells}

To assess the response to imino sugars, the S529V and S619R mutant GAAs generated by site-directed mutagenesis were transiently expressed in $\mathrm{CHO}-\mathrm{K} 1$ cells.

The cells were cultured in medium containing various concentrations $(0,10,50$ and $300 \mu \mathrm{M})$ of DNJ, NB-DNJ or NSA for $72 \mathrm{~h}$, and then the GAA activity in the cells was measured. The results are shown in Figure 2.

DNJ treatment apparently increased the enzyme activity of the S529V mutant GAA, as in the case of the wild type, with DNJ at 10 and $50 \mu \mathrm{M}$, although a high concentration $(300 \mu \mathrm{M})$ of DNJ rather decreased the enzyme activity. No response was observed for the S619R mutant GAA.

As to NB-DNJ and NSA, only the S529V mutant GAA responded to them, at 10 and $50 \mu \mathrm{M}$, but these chemicals decreased the enzyme
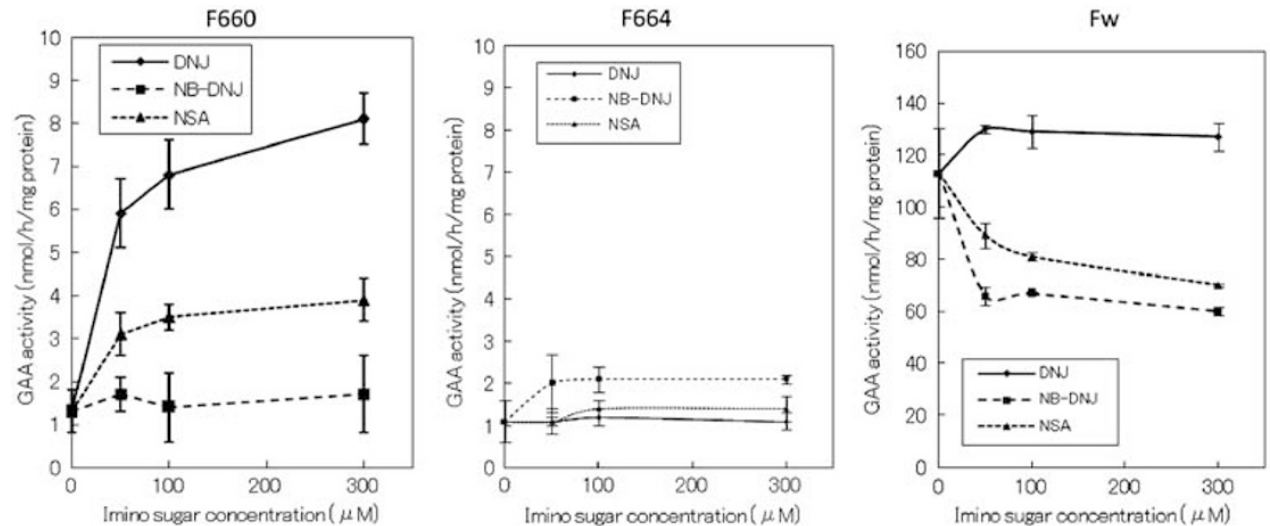

Figure 1 Effects of imino sugars on acid $\alpha$-glucosidase (GAA) activity of primary Pompe fibroblasts. F660 (genotype, p.S529V/p.S619R), F664 (p.R437C/ p.R437C) and Fw (wild type/wild type) were cultured for $24 \mathrm{~h}$ in medium containing various concentrations of 1-deoxynojirimycin (DNJ), $\mathrm{N}$-butyldeoxynojirimycin (NB-DNJ) or nojirimycin-1-sulfonic acid (NSA). Then, GAA activity in the cells was measured. 

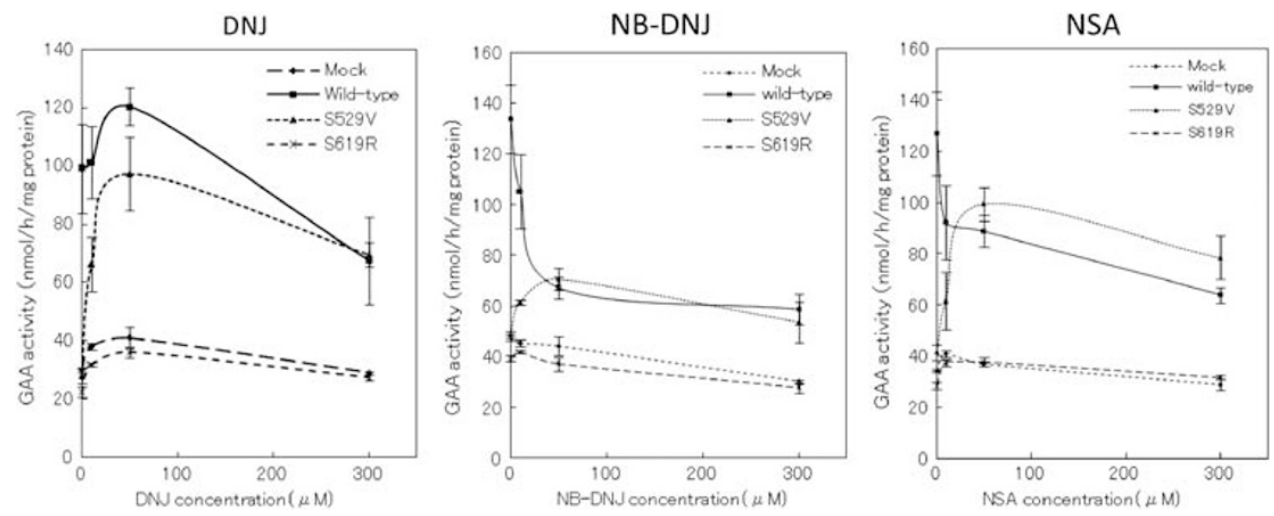

Figure 2 Effects of imino sugars on enzyme activity of acid $\alpha$-glucosidase (GAA) proteins expressed in Chinese hamster ovary (CHO) cells. The S529V and S619R mutant GAAs, and the wild-type one were expressed in CHO-K1 cells, and then the effects of imino sugars including 1-deoxynojirimycin (DNJ), $\mathrm{N}$-butyl-deoxynojirimycin (NB-DNJ) and nojirimycin-1-sulfonic acid (NSA) on the enzyme activities were examined.

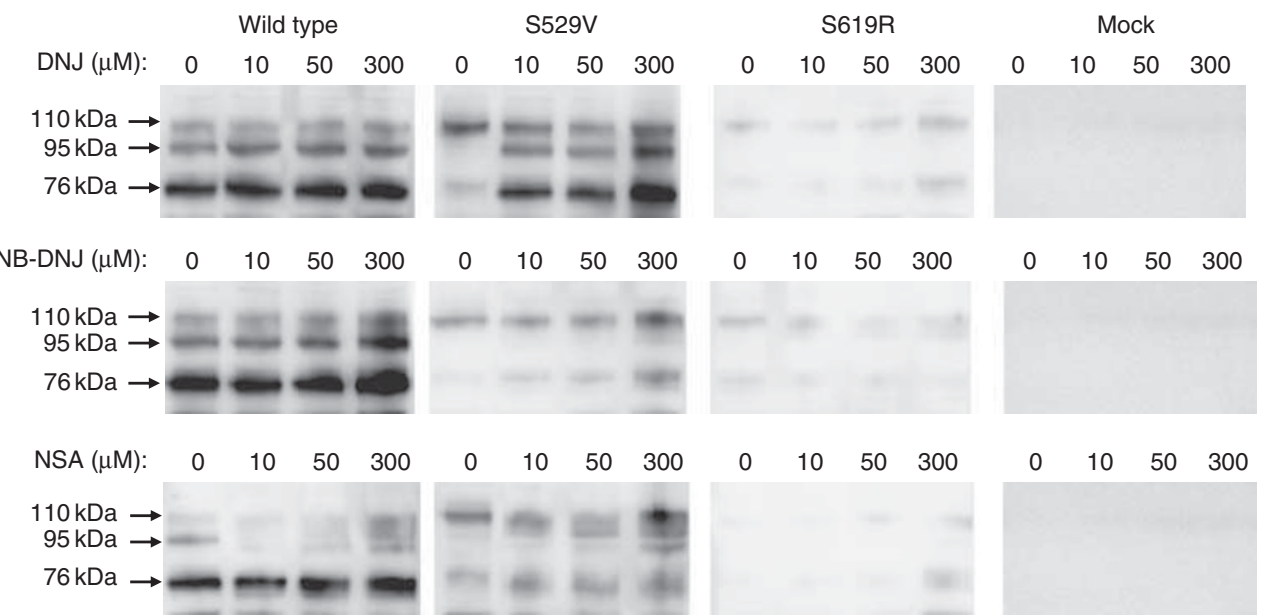

Figure 3 Western blotting profiles for acid $\alpha$-glucosidases (GAAs) expressed in CHO-K1 cells and effects of imino sugars on them. The S529V and S619R mutant GAAs, and the wild type one were expressed in $\mathrm{CHO}-\mathrm{K} 1$ cells, and then the cells were treated with 1-deoxynojirimycin (DNJ), $\mathrm{N}$-butyl-deoxynojirimycin (NB-DNJ) or nojirimycin-1-sulfonic acid (NSA), followed by western blotting analysis.

activity of the wild-type GAA. The S619R mutant GAA did not exhibit any response to them.

To determine whether these imino sugars improve the stability and/ or processing of the mutant GAAs or not, western blotting analysis was performed. The results are shown in Figure 3. The $110 \mathrm{kDa}$ precursor isozyme was detectable in all untreated cells. However, the $76 \mathrm{kDa}$ mature isozyme was not or only faintly detected for the S529V and S619R mutant GAAs, although it was clearly detectable in the case of the wild type. The total amounts of GAA protein as well as the $95 \mathrm{kDa}$ intermediate and $76 \mathrm{kDa}$ mature isozymes were increased by treatment with these imino sugars for the S529V mutant GAA, as in the case of the wild-type one. The S619R mutant GAA did not show any response to the imino sugars.

These results suggest that imino sugars including DNJ facilitate the transportation and processing of the S529V mutant GAA and stabilize it, resulting in an increase in GAA activity in cells.

\section{Structural change in GAA for each amino acid substitution}

Human GAA is composed of five domains; a trefoil type-P domain, an $N$-terminal $\beta$-sandwich domain, a $(\beta / \alpha)_{8}$-barrel domain, which includes catalytic residues D518 and D619 as the nucleophile and acid/base, respectively, with two inserted loops, a proximal C-terminal domain and a distal C-terminal domain, as reported previously.
Table 2 Structural changes in the GAA proteins

\begin{tabular}{lcccc}
\hline & \multicolumn{2}{c}{ Number of affected atoms } & & \\
\cline { 2 - 3 } Amino acid substitution & Main chain & Side chain & RMSD $\left(\AA^{\circ}\right)$ & ASA ( $\left(^{2}\right)$ \\
\hline S529V & 25 & 30 & 0.031 & 14 \\
S619R & 119 & 127 & 0.110 & 11 \\
\hline
\end{tabular}

Abbreviations: ASA, accessible surface area; GAA, acid $\alpha$-glucosidase; RMSD, root mean square distance.

To determine the structural changes in GAA for S529V and S619R, we constructed structural models of them. Then, the number of atoms affected by the amino acid substitution was determined for each model, and the root mean square distance and ASA values were calculated (Table 2), followed by coloring of the affected atoms (Figure 4).

S529 is located in the first loop in the $(\beta / \alpha)_{8}$-barrel domain, which suggests that the S529V amino acid substitution causes a moderate conformational change near the surface of the molecule far from the active site.

On the other hand, S619 is located in the $(\beta / \alpha)_{8}$-barell domain, which suggests that the S619R amino acid substitution leads to a large 

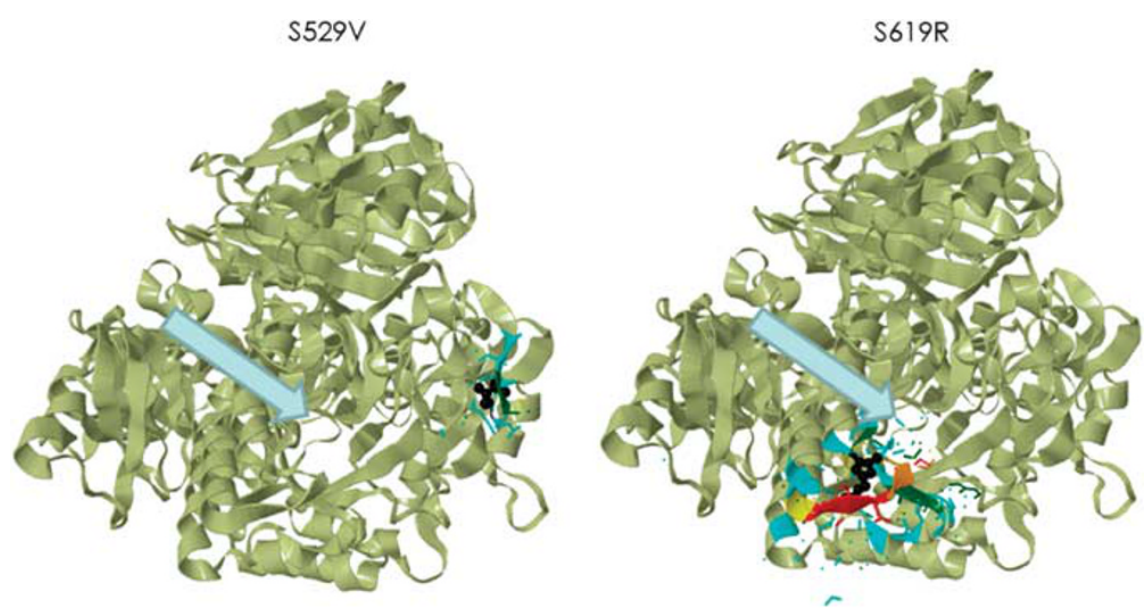

Figure 4 Coloring of the influenced atoms in the three-dimensional structures of the mutant acid $\alpha$-glucosidases (GAAs). The distributions of the influenced atoms in the S529V and S619R mutant GAAs are shown. Each atom is colored according to the distance between the atom in the mutant structure and the corresponding one in the wild type. The colors show the distance, as follows: light green $<0.15 \AA, 0.15 \AA \leqslant c y a n<0.30 \AA, 0.30 \AA \leqslant$ green $<0.45 \AA$, $0.45 \AA$ yellow, $0.60 \AA \leqslant$ orange $<0.75 \AA$, and red $\geqslant 0.75 \AA$. The total structure is shown as a ribbon model, and the atoms comprising a substituted amino acid are shown in black. Arrows indicate the active site.

a

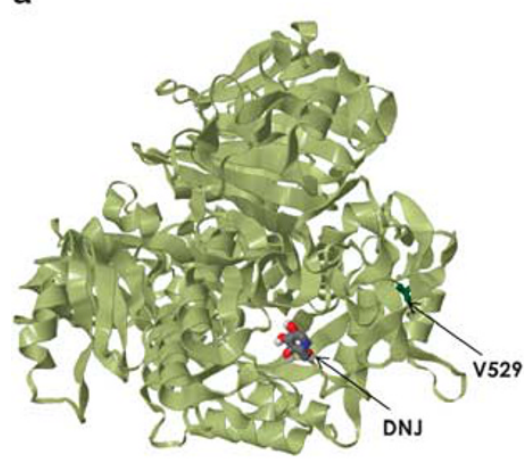

b

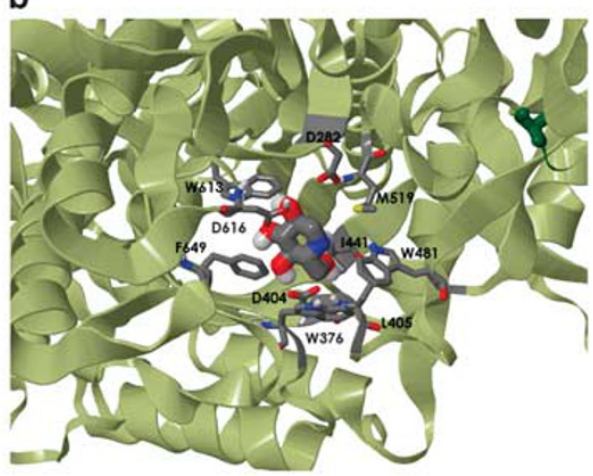

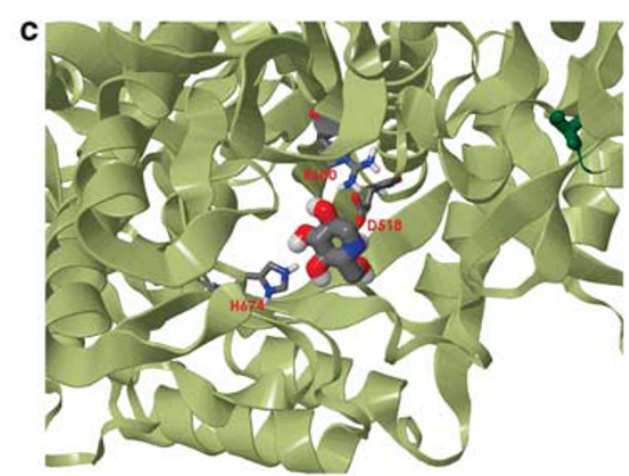

Figure 5 Docking model of the S529V mutant acid $\alpha$-glucosidase (GAA)/1-deoxynojirimycin (DNJ) complex. (a) Overview: locations of DNJ (stick model) and V529 (green), (b) DNJ and amino acid residues that are in van der Waals contact with DNJ, and (c) DNJ and amino acid residues that form hydrogen bonds with DNJ. Each backbone is shown as a light green ribbon model. The substituted amino acid is shown in green.

conformational change of the molecule and thereby affects the active site pocket structure.

Structural model of the complex of the S529V mutant GAA with DNJ bound to its active site

To elucidate the interaction between DNJ and the S529V mutant GAA, a structural model of DNJ bound to the active site of the mutant enzyme was built. Figure 5a shows the docking structure of the mutant enzyme protein and DNJ complex. Residues D282, W376, D404, D405, I441, W481, M519, W613, D616 and F649 of the enzyme molecule are thought to be in van der Waals contact with DNJ (Figure 5b). Furthermore, residues D518, R600 and H674 are predicted to form hydrogen bonds with DNJ (Figure 5c). DNJ almost completely occupies the active site pocket. 


\section{DISCUSSION}

Recently, pharmacological chaperone therapy has been investigated as a potential treatment for Pompe disease. This therapy is based on the concept that imino sugars including DNJ and NB-DNJ interact with mutant GAA proteins, and thereby improve their stability and transportation to lysosomes. ${ }^{13,14,28}$ However, these imino sugars are potent inhibitors of $\alpha$-glucosidases including GAA and intestinal maltase-glucoamylase, and the administration of an excess dose results in intracellular storage of glycogen, ${ }^{29}$ and glucosylated and galactosylated oligosaccharides. ${ }^{30}$ So, it seems to be difficult to determine the proper doses of these chemicals for pharmacological chaperone therapy, and inadequate doses of them would worsen Pompe disease and might lead to diarrhea. Furthermore, the mutant GAA species for which imino sugars are effective are limited. ${ }^{13,14,28}$ Hence, characterization of mutant GAAs responsive to imino sugars, and detailed information about the complex formation of a mutant GAA and an imino sugar are strongly required for improvement of pharmacological chaperone therapy.

As far as we know, there are about 80 Japanese Pompe patients. Out of them, at least 22 cases have been examined as to gene mutations, and five missense mutation species were identified in more than two Japanese Pompe families; p.S529V (4/44 alleles), p.R600C (8/44 alleles), p.S619R (6/44 alleles), p.D645E (3/44 alleles) and p.R672Q (3/44 alleles).

In this study, we examined the effect of DNJ on primary cultured fibroblasts obtained from 10 Japanese Pompe patients. Among them, only a strain carrying the p.S529V/p.S619R mutations responded to imino sugars. DNJ and NSA increased its enzyme activity approximately four- and twofold at $50 \mu \mathrm{M}$, although a high dose of the chemicals rather inhibited the enzyme activity. A previous study on a pseudodeficiency of GAA' revealed that residual enzyme activity, that is, $10-20 \%$ of the normal control level, could prevent glycogen storage. ${ }^{19}$ So, even a small increase in GAA activity on the administration of these imino sugars would be expected to improve the symptoms of Pompe disease. NB-DNJ did not show any apparent enhancement under the conditions examined here.

To determine which ones are responsive to imino sugars, mutant GAAs carrying the S529V and S619R amino acid substitutions were separately expressed in $\mathrm{CHO}-\mathrm{K} 1$ cells, and then the response to imino sugars was examined. The results revealed that only the S529V mutant GAA was responsive to DNJ, NB-DNJ and NSA. Considering the results of western blotting, these imino sugars should facilitate the transportation and processing of the S529V mutant GAA, and stabilize it. Recently, Flanagan et al. ${ }^{28}$ reported that 16 species of mutant GAAs, including p.S529V, responded to DNJ, and that 60 species including p.R600C, p.S619N, p.D645E and p.R672Q did not. Here we examined the characteristics of the S529V mutant GAA from a structural aspect. The results revealed that a small structural change occurred in the first loop in the $(\beta / \alpha)_{8}$-barrel domain, which did not affect the active site pocket. Even such a minor conformational change should be detected by the endoplasmic reticulum's quality control system and the mutant GAA should be quickly degraded before being transported to lysosomes.

We examined the interaction of the S529V mutant GAA with DNJ, which exhibited the strongest action on the mutant GAA, by building a structural model of the complex. DNJ should occupy the active site pocket, and bind strongly to amino acid residues comprising it through van der Waals contacts and hydrogen bonds.

For development of pharmacological chaperone therapy, it is important to confirm that the administration of an imino sugar reduces the glycogen content in a patient's cells. As glycogen does usually not accumulate in cultured Pompe fibroblasts, cultured myocytes derived from a Pompe patient carrying the mutation responsive to imino sugars would be suitable for the above purpose. Skeletal muscle tissue is one of the main tissues affected in Pompe disease, and there could be subtle tissue-specific differences in the glycogen metabolism and/or the modulation of the residual GAA activity between skeletal muscles and other tissues. Unfortunately, we could not obtain biopsied muscle tissues from the patient because the procedure is invasive. In the future, it is desirable to generate a Pompe mouse carrying the mutation responsive to imino sugars to examine the effects of chemicals on glycogen cleavage.

In conclusion, we examined the characteristics of the S529V mutant GAA responsive to imino sugars biochemically and structurally. The results provide us with a lot of information for improving the pharmacological chaperone therapy for Pompe disease.

\section{ACKNOWLEDGEMENTS}

We thank Dr J Ponder (Department of Biochemistry and Molecular Biophysics, Washington University) for providing us with the TINKER software. We also thank Ms Y Tanabe for typing the manuscript. This work was supported by Program for Research on Intractable Diseases of Health and Labor Research Grants (HS); the Program for Promotion of Fundamental Studies in Health Sciences of the National Institute of Biomedical Innovation (ID: 09-15, HS); the JAPS Asia/Africa Scientific Platform Program (HS); the Japan Society for the Promotion of Science (JAPS ID: 21390314, HS); and the High-Tech Research Center Project of the Ministry of Education, Culture, Sports, Science and Technology of Japan (S0801043, HS).

1 Hirschhorn, R. \& Reuser, A. J. J. in The Metabolic and Molecular Bases of Inherited Disease (eds Scriver,C. R., Beaudet, A. L., Sly, W. S., \& Valle, D.) 3389-3420 (McGraw-Hill, New York, 2001).

2 Hasilik, A. \& Neufeld, E. F. Biosynthesis of lysosomal enzyme in fibroblasts. Phosphorylation of mannose residues. J. Biol. Chem. 255, 4946-4950 (1980).

3 Moreland, R. J., Jin, X., Zhang, X. K., Decker, R. W., Lee, K. L., Cauthron, R. D. et al. Lysosomal acid $\alpha$-glucosidase consists of four different peptides processed from a single chain precursor. J. Biol. Chem. 280, 6780-6791 (2005).

4 Wisselaar, H. A., Kroos, M. A., Hermans, M. M. P., van Beeumen, J. \& Reuser, A. J. J. Structural and functional changes of lysosomal acid $\alpha$-glucosidase during intracellular transport and maturation. J. Biol. Chem. 268, 2223-2231 (1993).

5 Raben, N., Hill, V., Shea, L., Takikita, S., Baum, R., Mizushima, N. et al. Suppression of autophagy in skeletal muscle uncovers the accumulation of ubiquitinated proteins and their potential role in muscle damage in Pompe disease. Hum. Mol. Genet. 17, 3897-3908 (2008).

6 Hermans, M. M., van Leenen, D., Kroos, M. A., Beesley, C. E., Van Der Ploeg, A. T., Sakuraba, $H$. et al. Twenty-two novel mutations in the lysosomal $\alpha$-glucosidase gene (GAA) underscore the genotype-phenotype correlation in glycogen-storage disease type II. Hum. Mutat. 23, 47-56 (2004).

7 Montalvo, A. L., Bembi, B., Donnarumma, M., Filocamo, M., Parenti, G., Rossi, M. et al. Mutation profile of the GAA gene in 40 Italian patients with late onset glycogen storage disease type II. Hum. Mutat. 27, 999-1006 (2006).

8 Kroos, M., Pomponio, R. J., van Vliet, L., Palmer, R. E., Phipps, M., Van der Helm, R. et al. GAA Database Consortium, update of the Pompe disease mutation database with 107 sequence variants and a format for severity rating. Hum. Mutat. 29, e13-e26 (2008).

9 Ellgaard, L. \& Helenius, A. Quality control in the endoplasmic reticulum. Nat. Rev. Mol. Cell. Biol. 4, 181-191 (2003).

10 Kishnani, P. S., Nicolino, M., Voit, T., Rogers, R. C., Tsai, A. C., Waterson, J. et al. Chinese hamster ovary cell-derived recombinant human acid $\alpha$-glucosidase in infantile-onset Pompe disease. J. Pediatr. 149, 89-97 (2006).

11 Raben, N., Fukuda, T., Gilbert, A. L., de Jong, D., Thurberg, B. L., Mattaliano, R. J. et al. Replacing acid $\alpha$-glucosidase in Pompe disease: recombinant and transgenic enzymes are equipotent, but neither completely clears glycogen from Type II muscle fibers. Mol. Ther. 11, 48-56 (2005).

12 Parenti, G. Treating lysosomal storage diseases with pharmacological chaperones: from concept to clinic. EMBO Mol. Med. 1, 268-279 (2009).

13 Okumiya, T., Kroos, M. A., Vliet, L. V., Takeuchi, H., Van der Ploeg, A. T. \& Reuser, A. J. Chemical chaperones improve transport and enhance stability of mutant $\alpha$-glucosidases in glycogen storage disease type II. Mol. Genet. Metab. 90, 49-57 (2007).

14 Parenti, G., Zuppaldi, A., Pittis, M. G., Tuzzi, M. R., Annunziata, I., Meroni, G. et al. Pharmacological enhancement of mutated $\alpha$-glucosidase activity in fibroblasts from patients with Pompe disease. Mol. Ther. 15, 508-514 (2007). 
15 Porto, C., Cardone, M., Fontana, F., Rossi, B., Tuzzi, M. R., Tarallo, A. et al. The pharmacological chaperone $\mathrm{N}$-butyldeoxynojirimycin enhances enzyme replacement therapy in Pompe disease fibroblasts. Mol. Ther. 17, 964-971 (2009).

16 Tsunoda, H., Ohshima, T., Tohyama, J., Sasaki, M., Sakuragawa, N. \& Martiniuk, F. Acid $\alpha$-glucosidase deficiency: identification and expression of a missense mutation (S529V) in a Japanese adult phenotype. Hum. Genet. 97, 496-499 (1996).

17 Kanazawa, N., Miyamoto, T., Ihara, K., Miyoshi, Y., Sakai, N., Inui, K. et al. Novel mutation and a frequent mutation in Japanese juvenile patients with acid maltase deficiency. J. Inher. Metab. Dis. 26, 310 (2003).

18 Tsujino, S., Huie, M., Kanazawa, N., Sugie, H., Goto, Y., Kawai, M. et al. Frequent mutations in Japanese patients with acid maltase deficiency. Neuromuscul. Disord. 10, 599-603 (2000).

19 Tajima, Y., Matsuzawa, F., Aikawa, S., Okumiya, T., Yoshimizu, M., Tsukimura, T. et al. Structural and biochemical studies on Pompe disease and a 'pseudodeficiency of acid alpha-glucosidase'. J. Hum. Genet. 52, 898-906 (2007).

20 Tajima, Y., Kawashima, I., Tsukimura, T., Sugawara, K., Kuroda, M., Suzuki, T. et al. Use of a modified $\alpha$-N-acetylgalactosaminidase in the development of enzyme replacement therapy for Fabry disease. Am. J. Hum. Genet. 85, 569-580 (2009).

21 Sugawara, K., Saito, S., Sekijima, M., Ohno, K., Tajima, Y., Kroos, M. A et al. Structural modeling of mutant $\alpha$-glucosidases resulting in a processing/transport defect in Pompe disease. J. Hum. Genet. 54, 324-330 (2009).

22 Dudek, M. J. \& Ponder, J. W. Accurate modeling of the intramolecular electrostatic energy of proteins. J. Comput. Chem. 16, 791-816 (1995).
23 Ren, P. \& Ponder, J. W. Polarizable atomic multipole water model for molecular mechanics simulation. J. Phys. Chem. B. 107, 5933-5947 (2003).

24 Sugawara, K., Ohno, K., Saito, S. \& Sakuraba, H. Structural characterization of mutant $\alpha$-galactosidases causing Fabry disease. J. Hum. Genet. 53, 812-814 (2008).

25 McDonald, I. K. \& Thornton, J. M. Satisfying hydrogen bonding potential in proteins. J. Mol. Biol. 238, 777-793 (1994).

26 Yoshimizu, M., Tajima, Y., Matsuzawa, F., Aikawa, S., Iwamoto, K., Kobayashi, T. et al. Binding parameters and thermodynamics of the interaction of imino sugars with a recombinant human acid $\alpha$-glucosidase (alglucosidase alfa): Insight into the complex formation mechanism. Clin. Chim. Acta. 391, 68-73 (2008).

27 Sugawara, K., Tajima, Y., Kawashima, I., Tsukimura, T., Saito, S., Ohno, K. et al. Molecular interaction of imino sugars with human $\alpha$-galactosidase: insight into the mechanism of complex formation and pharmacological chaperone action in Fabry disease. Mol. Genet. Metab. 96, 233-238 (2009).

28 Flanagan, J. J., Rossi, B., Tang, K., Wu, X., Mascioli, K., Donaudy, F. et al. The pharmacological chaperone 1-deoxynojirimycin increases the activity and lysosomal trafficking of multiple mutant forms of acid alpha-glucosidase. Hum. Mutat. 30, 1683-1692 (2009).

29 Wisselaar, H. A., van Dongen, J. M. \& Reuser, A. J. J. Effects of $\mathrm{N}$-hydroxyethyl1-deoxynojirimycin (BAY $m$ 1099) on the activity of neutral- and acid alpha-glucosidases in human fibroblasts and HepG2 cells. Clin. Chim. Acta 182, 41-52 (1989).

30 Boomkamp, S. D., Rountree, J. S. S., Neville, D. C. A., Dwek, R. A., Fleet, G. W. J. \& Butters, T. D. Lysosomal storage of oligosaccharide and glycosphingolipid in imino sugar treated cells. Glycoconj. J. 27, 297-308 (2010). 